\title{
Recurrent Bacterial Meningitis: A New Reason to Wear a Seat Belt While Driving
}

\author{
Attila S. Nemeth, mo ${ }^{1}$ \\ Robert E. Crook, MD ${ }^{1}$ \\ Sandra Glagola, $0^{1}$ \\ Raja Shekhar R. Sappati \\ Biyyani, $\mathrm{MD}^{2}$
}

\author{
${ }^{1}$ Department of Medicine, Case Western Reserve University at MetroHealth Medical Center, Cleveland, Ohio. \\ ${ }^{2}$ Division of Hospital Medicine, Department of Medicine, Case Western Reserve University at \\ MetroHealth Medical Center, Cleveland, $\mathrm{OH}$.
}

Disclosure: Nothing to report.

Journal of Hospital Medicine 2010;5:E1-E3. @ 2010 Society of Hospital Medicine.

KEYWORDS: diagnostic decision making, multi-disciplinary care, bacterial meningitis.

Recurrent bacterial meningitis (RBM), particularly when caused by Streptococcus pneumoniae, warrants an aggressive and thorough evaluation to exclude transdural communication. We present an unusual case of RBM as a late manifestation of a traumatic head injury sustained 10 years prior and describe presentation, etiology, diagnosis, and treatment options for RBM.

\section{Case Report}

A middle-aged woman with type 2 diabetes mellitus, hypertension, and a prior history of $S$. pneumoniae meningitis 1 year earlier, presented to an outside hospital with complaints of fever, headache, and change in mental status. Materials for basic laboratory tests and blood cultures were drawn in the Emergency Department; these showed diabetic ketoacidosis. Computed tomography (CT) scan of the head was negative and a lumbar puncture (LP) was attempted, but was unsuccessful. The patient was started on intravenous insulin drip, vancomycin, and ceftriaxone and was transported to our facility via Life-Flight. She also developed acute respiratory failure requiring mechanical ventilation.

After arrival, the patient had a normal repeat CT scan of her head and a successful LP. Cerebrospinal fluid (CSF) revealed 9064 white blood cells (WBCs) $/ \mathrm{mm}^{3}$ with $77 \%$ neutrophils and 9\% lymphocytes, protein concentration of 275 $\mathrm{mg} / \mathrm{dL}$, and glucose of $93 \mathrm{mg} / \mathrm{dL}$. CSF culture and Gram stain were negative, while 1 blood culture drawn at the outside hospital grew penicillin-resistant S. pneumoniae (MIC $2 \mu \mathrm{g}$ / $\mathrm{mL}$ ). WBC count was $9660 / \mathrm{mm}^{3}$ with $45 \%$ band forms. Bacterial meningitis was confirmed and the patient was continued on intravenous antibiotics and insulin drip. Additional laboratory studies revealed normal complement levels and a negative human immunodeficiency virus (HIV) 1 and HIV 2 antibody screen. The patient was extubated in 48 hours. and was treated with a total of 2 weeks of ceftriaxone and vancomycin for penicillin-resistant $S$. pneumoniae meningitis.

The patient had an uneventful full recovery and was discharged from the hospital with neurosurgery follow-up. The neurosurgeon ordered a CT scan of the facial bones, which revealed an irregular calcification in the right frontal sinus adjacent to the cribriform plate and thinning of the posterior wall of the sinus. Upon requestioning at a subsequent neurosurgical appointment, the patient recalled being an unrestrained passenger and striking her head against the windshield in a motor vehicle accident (MVA) approximately 10 years ago. Ever since the MVA, she noticed intermittent postnasal discharge while recumbent. However, she never sought a medical opinion and denied complaints of anterior rhinorrhea.

A CT cisternography confirmed the presence of CSF leakage with contrast accumulation via a defect in the right paramedian cribriform plate. Contrast opacification was seen in the fovea ethmoidalis extending into the right frontal sinus (Figure 1). The patient subsequently underwent transnasal endoscopic CSF leak repair (Figure 2). The postoperative cisternogram did not reveal the transdural communication. However a follow-up cisternogram performed 3 months later demonstrated a recurrent CSF leak.

The patient was rehospitalized with grand-mal seizures and a third episode of $S$. pneumoniae meningitis, this time with a penicillin-sensitive strain. She was treated with a 2week course of ceftriaxone and also received heptavalent pneumococcal vaccine to supplement the 23-valent pneumococcal vaccine. Two weeks after the hospital discharge, the patient underwent successful bifrontal transcranial repair. Currently, she has been disease-free for 3 months and is followed closely by neurosurgery as an outpatient.

\section{Discussion}

After immune deficiency is ruled out, it is essential to evaluate for transdural communication between the subarachnoid space and the base of the skull resulting in a CSF leak as a cause for RBM. Meningitis secondary to a CSF leak is most commonly caused by $S$. pneumoniae, followed by Neisseria meningitidis and Haemophilus influenzae. ${ }^{1}$ Complement and immunoglobulin subclass defects may also predispose to RBM. ${ }^{2,3}$

A recent case series by Adriani et al. ${ }^{4}$ suggested that as many as $77 \%$ of patients with RBM have an identifiable risk factor such as a remote head injury or CSF leakage. CSF 


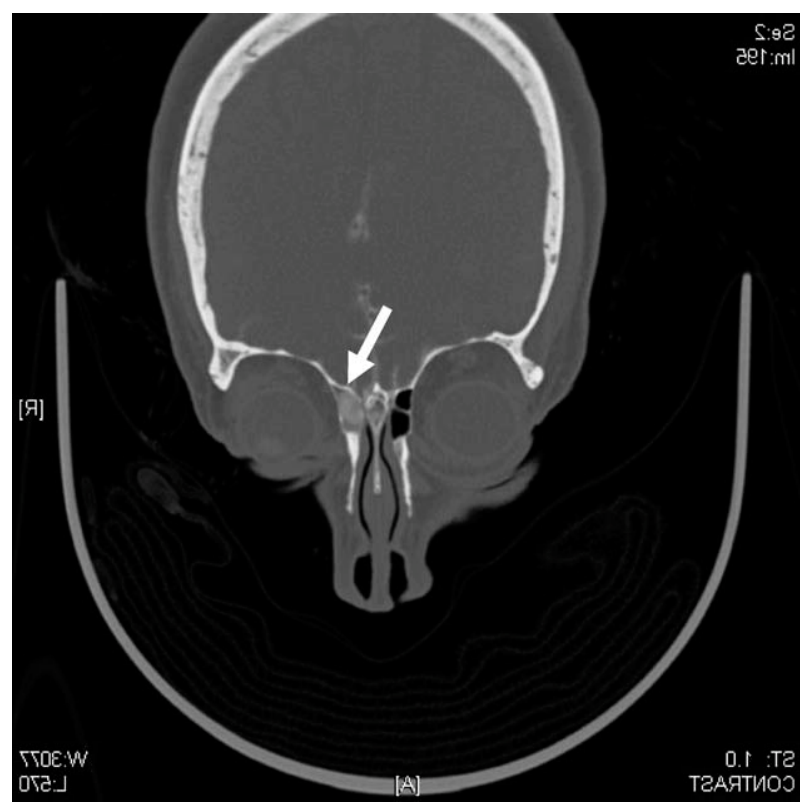

FIGURE 1. Computed tomography cisternography confirms the presence of CSF leakage as contrast accumulation is noted via a defect in the right paramedian cribriform plate (arrow). Contrast opacification is also noted in the fovea ethmoidalis extending into the right frontal sinus.

rhinorrhea is most often secondary to trauma, occurring in approximately $1 \%$ to $3 \%$ of all blunt head injuries. ${ }^{2}$ Accidental falls, MVAs, altercations, and gunshot wounds are also commonly responsible. ${ }^{3}$ Nontraumatic CSF leaks are very rare but may be secondary to spontaneous, congenital, or iatrogenic etiologies. ${ }^{1,3}$ Spontaneous CSF leaks could also occur due to violent sneezing or coughing., ${ }^{1,3}$ Congenital defects include weakened preformed pathways, failure of germ layer closures, and bone imperfections. ${ }^{1,3}$ Infrequently, CSF leak can be a complication of intracranial, otologic, nasal, or paranasal sinus surgeries. ${ }^{1,3}$ Other rarer etiologies include intracranial tumors and hydrocephalus. ${ }^{1,3}$

Bacterial meningitis due to traumatic CSF leak can present within 24 hours to as long as several decades after the development of the leak. ${ }^{2,3}$ Along with the classic symptoms and signs of meningitis, including fever, headache, neck stiffness, change in sensorium, seizures, and vomiting, patients may also present with CSF rhinorrhea, CSF otorrhea, hearing impairment, or cranial injury residua. ${ }^{3,5}$ It is important to note that CSF rhinorrhea and otorrhea are not always present in cases of chronic, posttraumatic CSF leaks.

The visualization of a fracture or bony dehiscence is very difficult but critical for identification and surgical repair. Frontal and ethmoid sinuses and cribriform plate are common fracture sites. ${ }^{1,3,5}$ CSF leakage may be from the anterior, middle, or posterior compartments, eventually ending in the nasal cavity. ${ }^{1,3,5}$ Various imaging modalities, including contrast cisternogram, high-resolution CT, fluorescein nasal endoscopy, and magnetic resonance imaging (MRI) have

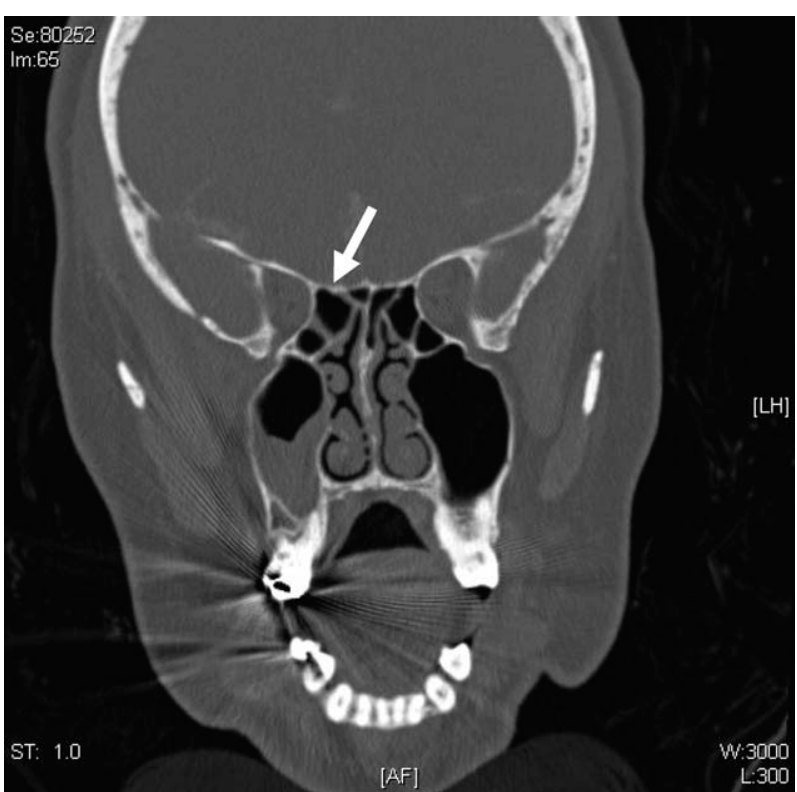

FIGURE 2. Repeat computed tomography cisternogram following surgical repair of CSF leak does not reveal residual extravasation of contrast from the subarachnoid space into the frontal or ethmoid sinuses (arrow).

been advocated for diagnosing the source of CSF leak with variable sensitivity and specificity. ${ }^{6}$ High-resolution CT helps in identifying surgical anatomy and bony defects whereas contrast cisternography is confirmatory when the CSF leak is active. ${ }^{1,6}$ Protein electrophoresis demonstrating 2 electrophoretically separate transferrin bands confirms CSF. ${ }^{7}$

In patients with persistent CSF rhinorrhea, there is a $19 \%$ overall risk of meningitis with an annual incidence of 0.3 meningitis episodes per year. ${ }^{8}$ The risk of meningitis is the greatest in the first year following the onset of a CSF leak. ${ }^{8}$ Generally, patients with posttraumatic CSF leak lasting more than 7 to 10 days need surgical repair to decrease their risk of bacterial meningitis. ${ }^{1,3,5}$ Endoscopic surgical correction with a success rate of $90 \%$ is an effective treatment for CSF leak and involves placement of a temporary lumbar drain in addition to endonasal duraplasty performed with the aid of microscope and/or nasoscope. ${ }^{9}$ Complicated anterior cranial base fractures are not as amenable to endoscopic repair and may require a combined intracranial extradural and intradural approach or a transcranial approach. ${ }^{10}$

Information on prophylactic antibiotics and vaccination is still evolving. Currently available evidence from randomized control trials does not support prophylactic antibiotic use in patients with basilar skull fracture with or without the evidence of CSF leakage. ${ }^{11}$ A meta-analysis of 4 randomized controlled trials of patients with acute basilar skull fracture showed no significant difference between the antibiotic prophylaxis groups and control groups with respect to reduction of the frequency of meningitis, the need for surgical correction, meningitis-related mortality, and all-cause 
mortality. ${ }^{11}$ Direct invasion of the meninges by nasopharyngeal bacteria bypassing the circulating serum antibodies may limit the potential effectiveness of vaccination in preventing RBM. ${ }^{12}$ However, vaccination is generally recommended in patients with complement or immunoglobulin deficiency or after splenectomy. ${ }^{4}$

\section{Address for correspondence and reprint requests:}

Raja Shekhar R. Sappati Biyyani, MD, MetroHealth Medical Center, 2500 MetroHealth Dr., Cleveland, OH 44109-1998; Telephone: 216-778-

5162; Fax: 216-778-4105; E-mail: rsappati@metrohealth.org Received 20 May 2008; revision received 14 January 2009; accepted 18 January 2009.

\section{References}

1. Pappas DG Jr, Hammerschlag PE. Cerebrospinal fluid rhinorrhea and recurrent meningitis. Clin Infect Dis. 1993;17:364-368.

2. Schick B, Weber R, Kahle G, et al. Late manifestations of traumatic lesions of the anterior skull base. Skull Base Surg. 1997;7(2):77-83.

3. Hand LW, Sanford JP. Posttraumatic bacterial meningitis. Ann Intern Med. 1970;72:869-874.
4. Adriani KS, van de Beek D, Brouwer MC, et al. Community-acquired recurrent bacterial meningitis in adults. Clin Infect Dis. 2007;45: e46-e51.

5. Applelbaum E. Meningitis following trauma to the head and face. JAMA. 1960;173:1818-1822.

6. Lloyd MNH, Kimber PM, Burrows EH. Post-traumatic cerebrospinal fluid rhinorrhea: modern high-definition computed tomography is all that is required for the effective demonstration of the site of leakage. Clin Radiol. 1994;49:100-103.

7. Irjala K, Suonpää J, Laurent B. Identification of CSF leakage by immunofixation. Arch Otolarygol. 1979;105:447-448.

8. Daudia A, Biswas D, Jones NS. Risk of meningitis with cerebrospinal fluid rhinorrhea. Ann Otol Rhinol Laryngol. 2007;116 (12):902-905.

9. McMains KC, Gross CW, Kountakis SE. Endoscopic management of cerebrospinal fluid rhinorrhea. Laryngoscope. 2004;114(10):1833-1837.

10. Scholsem M, Scholtes F, Collignon F, et al. Surgical management of anterior cranial base fractures with cerebrospinal fluid fistulae: a single-institution experience. Neurosurgery. 2008;62:463-471.

11. Ratilal B, Costa J, Sampaio C. Antibiotic prophylaxis for preventing meningitis in patients with basilar skull fractures. Cochrane Database Syst Rev. 2006;25(1):CD004884.

12. Davachi F, Bregu H, Lito G. Recurrent streptococcus pneumoniae meningitis. J Trop Pediatr. 2002;48:249-250. 